\title{
Nature of Energy Interactions
}

\author{
Cynelle Olívia de Souza
}

\begin{abstract}
Electromagnetic waves have an electric and magnetic field. There is an intimate relationship between these two fields and in this present work we adopt the theory that subatomic particles, here being considered neutrinos, can carry the thermal energy calculated through intrinsic kinetic energy. The field of neutrinos determined here may be responsible for the formation of the second harmonic, recently discovered, in which highly energetic electromagnetic waves, in the order of terahertz, excite superconducting electrons. Theories related to the electron and the electric and magnetic fields were also addressed, as their nature is fundamental to the understanding of electromagnetic waves, the interaction between them and the broad effects on nature.
\end{abstract}

Keywords:- Strings-Energies-Interactions-Wave.

\section{INTRODUCTION}

For decades, electromagnetic waves have aroused and aroused human curiosity about their nature, their effects and mysteries.

With the explosion of highly concentrated energy, tiny filaments of energy, with variation of energy inside, started to move intrinsically and electromagnetically, producing frequencies of various characteristics, in addition to small magnetic poles in their outermost regions. When agglomerating to form larger particles, the fields produced are reduced, but concentrated. With the larger mass of matter, zones of lesser energy appear on its periphery. It is due to these zones that gravity occurs, becoming one of the fundamental forces of the universe.
In the nucleus of atoms, neutrons attract each other and protons so that between materials there are different levels of energy that attract each other.

The force required to keep the nucleus cohesive is fundamental to the stability of matter.

The nuclear force also acts between two neutrons, as well as between a proton and a neutron. It is then that it guarantees the stability of the nucleus. That is why it is so difficult to tear protons and neutrons out of the nucleus of an atom. It is easier to pluck electrons.

"Atnuclear physics, nuclear force is the force that occurs betweencores(protonsandneutrons) ofatomic nucleus. This interaction is responsible for the cohesion between the differentparticlesthat make them up. Neutrons do not haveelectric charge, while protons have a positive charge. Strong nuclear interaction overcomesrepulsionprotons, positively charged, avoiding their dispersion. Usually the number of nuclei per $\mathrm{A}$ is represented; the number of protons per $Z$ and the number of neutrons per $\mathrm{N}$, like this: $\mathrm{A}=\mathrm{Z}+\mathrm{N}$.

The electrons are attached to particles smaller than it with high energy and different frequencies, which causes it to oscillate in a region of space. This energy is stronger than the gravitational and electromagnetic energy exerted by the nucleus.

The two forces that act together are gravity and magnetism. The resulting energy, which includes the kinetics of the filaments of matter (as in electrons), produces a third form of energy; this may be responsible for chemical bonds by heat.

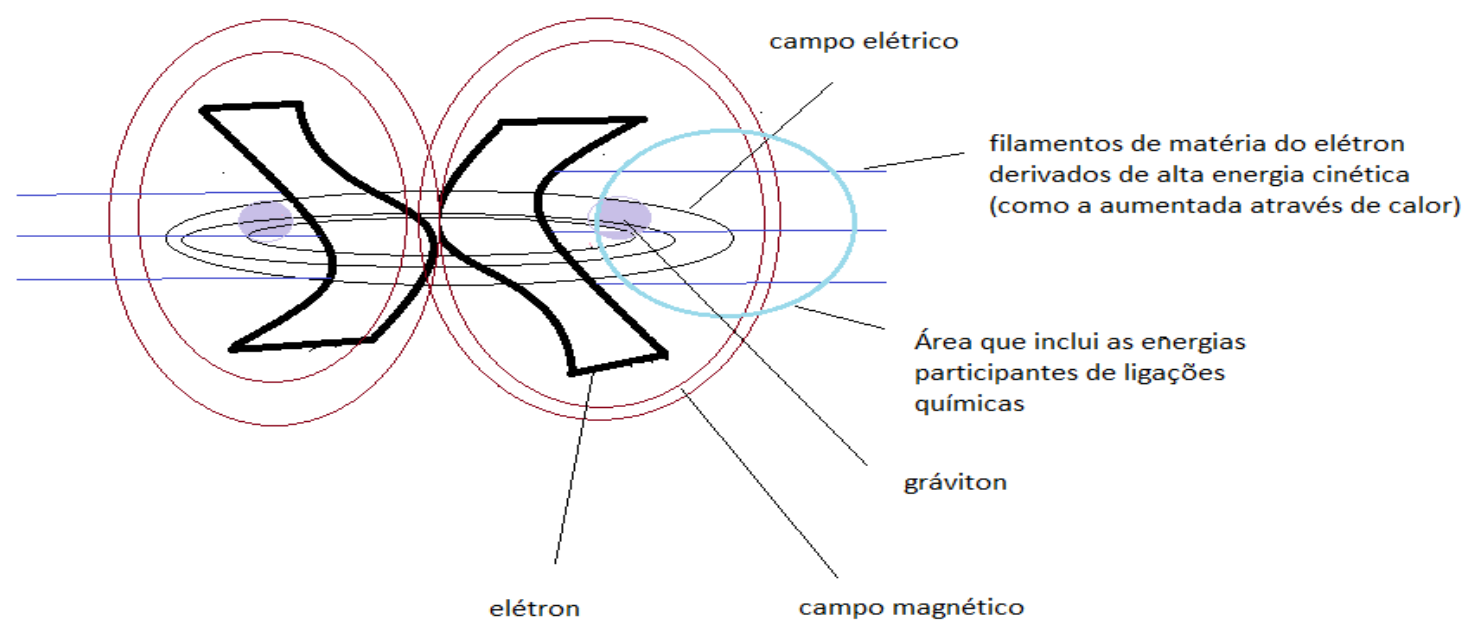

Fig 1:- electron and associated energies Source: own authorship 
ISSN No:-2456-2165

All matter, from its elementary state to the most complex one, presents a set of vibrations at different frequencies, amplitudes and even modulation (in which one wave overlaps the other to carry it). Pulse sequences can also occur. What causes one wave to not interfere with another is probably the cloud of neutrinos, which form a kind of barrier.

A team employed laser flashes at a rate of trillions of pulses per second, which helps to accelerate superconductors and therefore access new quantum states of matter. After tracking the light emitted by the accelerated electron pairs, they found light at twice the input frequency used to accelerate the electrons.

The second harmonic frequency may have been the result of kinetic energy present in the field of neutrinos associated with the photon.
"Finding ways to control, access and manipulate the special resources of the quantum world and connect them to real-world problems is a major scientific goal these days," said Perakis. Wang, in turn, stated that the study and development of technologies associated with this discovery will allow for high speeds and low energy consumption in future quantum and electronic computing strategies [1].

At lower frequencies, the neutrino field probably does not have enough energy to promote the photoelectric effect and thus induce the formation of a second harmonic.

Uma outra possivel evidencia de neutrinos em ondas eletromagneticas é a atração que as mesmas sofrem por buracos negros.

If there were no material that interacted gravitationally in the electromagnetic waves, the strong gravirational action would not exist.

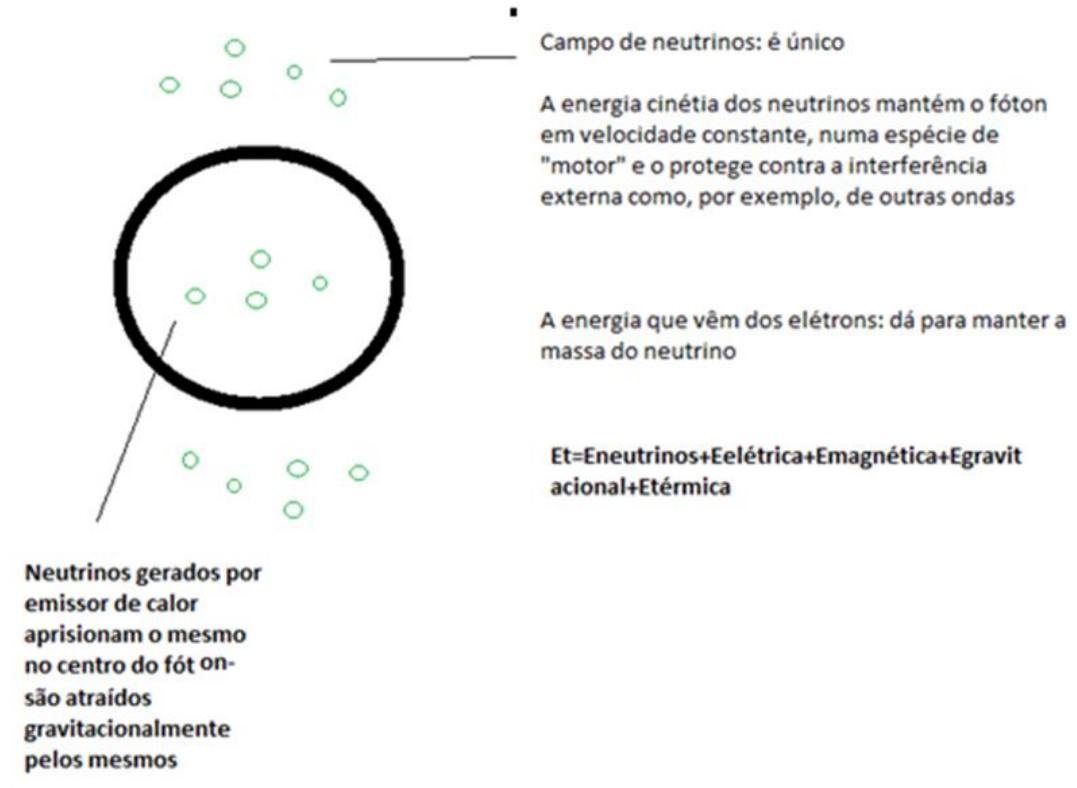

Fig 2:- Photons and associated components

Source: own author

\section{PROPERTIES OF ELECTROMAGNETIC WAVES}

Accelerated electrons emit photons that carry the magnetic and electric fields, at the speed of light, which is $300,000 \mathrm{~km} /$ second.

It is then possible to imagine light as being formed by several wave "packages", called photons, whose energy (E) depends on the frequency of the wave, according to the equation [2].

$\mathrm{E}=\mathrm{hf}$

However, there are other parameters to be analyzed when measuring the energy density of light, that is, its energy per volume unit [3]:
Total density of stored energy: $\varepsilon 0=\mathrm{E}^{2}$

$\mathrm{E}=$ electricity

Densities of electrical and magnetic energy:

$\mu \mathrm{E}(\overline{\mathrm{r}}, \mathrm{t})=1 / 2 \varepsilon 0 \mathrm{E}^{2}$

$\mu \mathrm{B}(\overline{\mathrm{r}}, \mathrm{t})=\mathrm{B}^{2} / 2 \mu 0$

Energy transport:

$\mathrm{S}=(1 / \mu 0)$. AND . B

$\mathrm{E} \times \mathrm{B}=\mu 0$. I

$\mathrm{I}=\mathrm{S}$

$\mathrm{I}=\Delta \mathrm{U} / \Delta \mathrm{a} \Delta \mathrm{t}$

$\mathrm{P}=\mathrm{dU} / \mathrm{dt}$ 


\section{TRANSPORT OF ELECTROMAGNETIC ENERGY}

The temporal transport of energy density is defined by an average, according to the graph below:

\section{TRANSPORTE DE ENERGIA}

A média temporal da densidade de energia é dada por

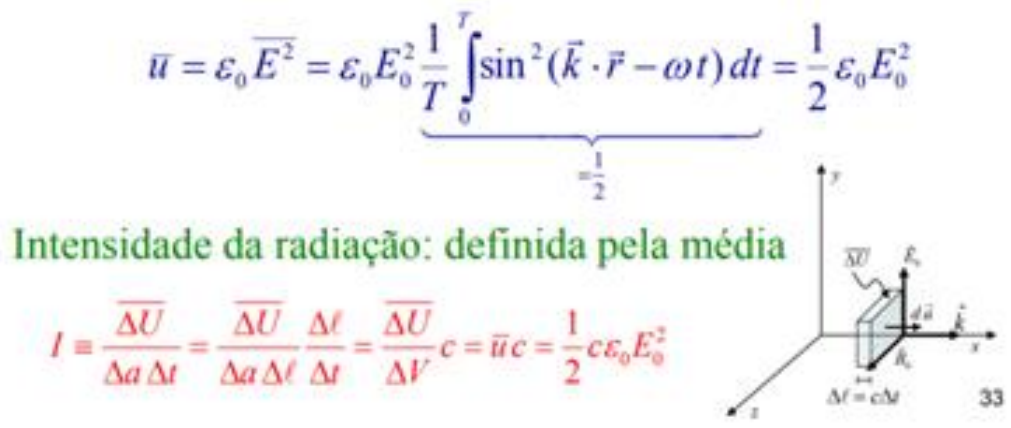

Graph 1:- Energy transport

Source: Electromagnetic waves [3]

That is, the average energy density can be considered:

$\mu \mathrm{c}=(1 / 2) \cdot c \cdot \mathrm{e} 0 \cdot \mathrm{E}^{2} 0$

The linear moment transport of the electromagnetic wave also occurs. Upon reaching an obstacle, a radiation pressure occurs:

$\mathrm{F} / \mathrm{A}=21 / \mathrm{c}$

\section{Transporte de momento linear : Pressão de radiação}

$$
\begin{aligned}
& \qquad \overline{\Delta U}=I A \Delta t \quad \Delta \vec{p}_{a}=\frac{\overline{\Delta U}}{c} \hat{k} \quad \Delta \vec{p}_{r}=2 \frac{\overline{\Delta U}}{c} \hat{k} \\
& \begin{array}{l}
\text { Pressão de radiação } \\
\text { na absorção total }
\end{array} F_{s}=\frac{\Delta p_{e}}{\Delta t}=\frac{I A}{c} \Rightarrow \text { Pressão }_{a t s}=\frac{F_{s}}{A}=\frac{I}{c} \\
& \begin{array}{l}
\text { Pressão de radiação } \\
\text { na reflexão total }
\end{array} F_{r}=\frac{\Delta p_{r}}{\Delta t}=\frac{2 I A}{c} \Rightarrow \text { Pressão }_{n f}=\frac{F_{r}}{A}=\frac{2 I}{c}
\end{aligned}
$$
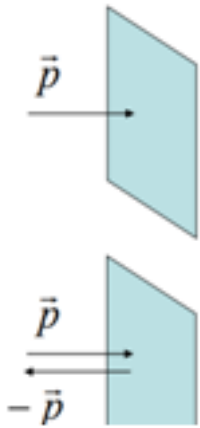

\section{Graph 2:- Linear moment transport: Radiation pressure}

Source: Electromagnetic waves [3]

The same element that carries the deltaU energy density also carries the linear moment, namely:

$S=(1 / \mu) \operatorname{ExB}$ 


\section{Transporte de momento linear: Pressão de radiação}

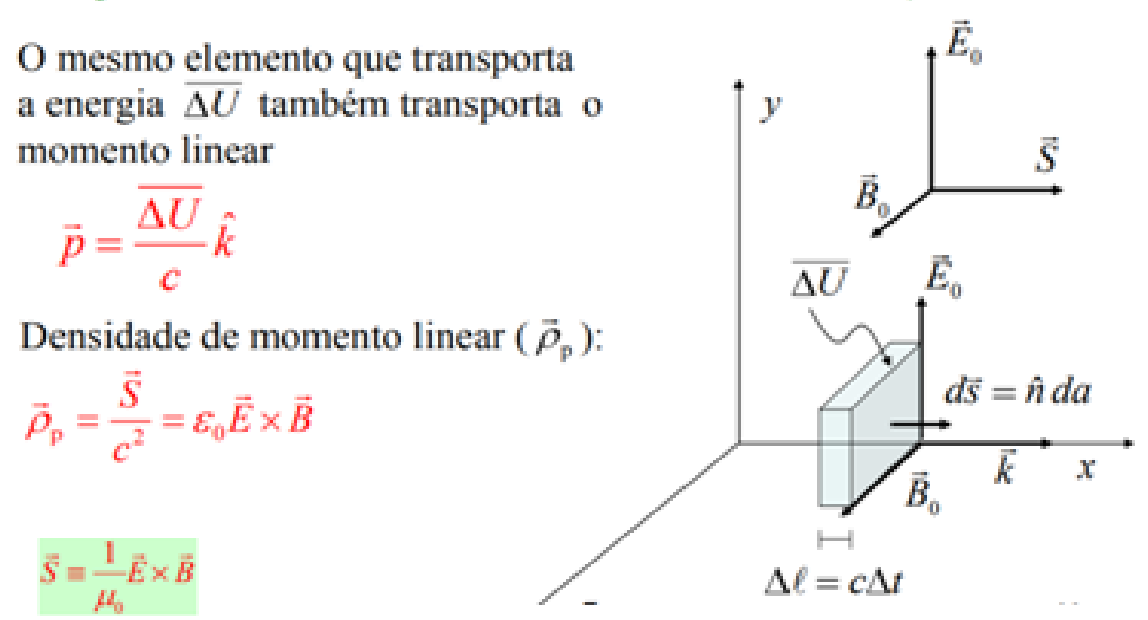

Graph 3:- Linear moment transport: Radiation pressure

Source: Electromagnetic waves [3]

The direction of $\mathrm{E} \times \mathrm{B}$ occurs in the direction of propagation of the wave.

Electromagnetic waves carry energy (S) and moment $\mathrm{p}$ (and therefore exert pressure $\mathrm{P}$ )

Electromagnetic waves can be polarized (linear, circular, elliptical) or non - polarized.

Some polarizing materials allow only the electric field component to pass parallel to the polarization axis [3].

\section{MATTER AND ENERGY}

With the explosion of highly concentrated energy, tiny energy filaments, with varying energy inside, started to move intrinsically and electromagnetically, producing frequencies of various characteristics, in addition to small magnetic poles in their outermost regions. By agglomerating to form larger particles, the fields produced are reduced, but concentrated. With the larger mass of matter, zones of lesser energy appear on its periphery. It is due to these zones that gravity occurs, becoming one of the fundamental forces of the universe.

In the nucleus of atoms, neutrons attract each other and protons so that between materials there are different levels of energy that attract each other.

The force required to keep the nucleus cohesive is fundamental to the stability of matter.
The nuclear force also acts between two neutrons, as well as between a proton and a neutron. It is then that it guarantees the stability of the nucleus. That is why it is so difficult to tear protons and neutrons out of the nucleus of an atom. It is easier to pluck electrons from the periphery. QUOTE [4].

The electrons are attached to particles smaller than it with high energy and different frequencies, which causes it to oscillate in a region of space. This energy is stronger than the gravitational and electromagnetic energy exerted by the nucleus.

String Theory can be consistent with many aspects of the quantum world, especially with regard to the minimum frequencies exerted by the same energy filament, in the same way that it occurs with enzymes and other proteins.

The frequency of vibration would function as "energy addresses", causing changes, even if minimal, in the fundamental fields - gravitational, electric and magnetic. The energy derived from thermal radiation would favor chemical interactions such as breaks or bonds; this would be directly dependent on the binding frequency of the molecules. An example: molecules with a weak, covalent chemical bond can easily break when thermal energy accelerates their paired electrons. Molecules with resonant frequencies require less energy and work to "break" the connections. 


\section{COMPOSITION OF ELECTRICAL LOADS}

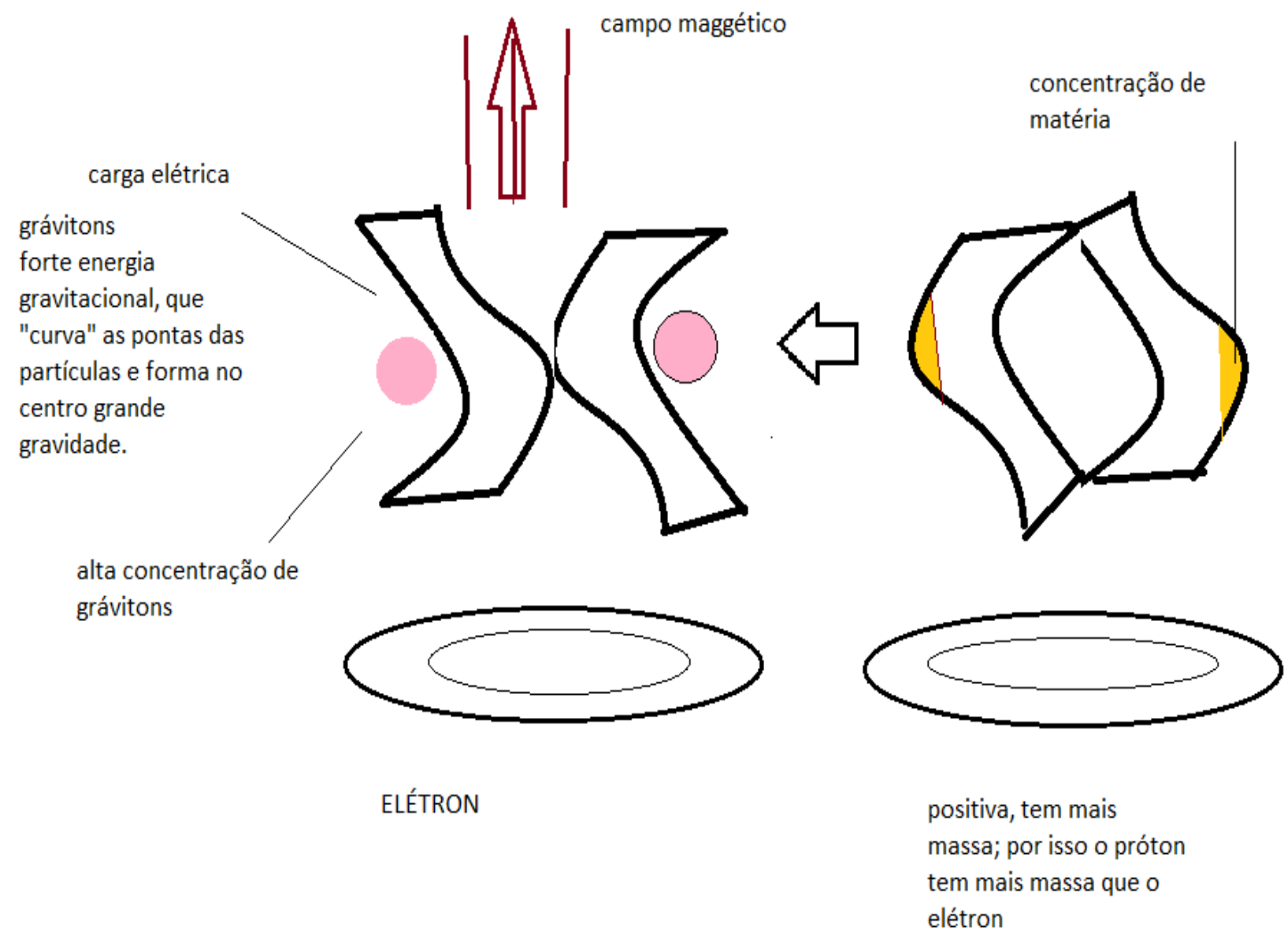

Fig 3:- Composition of negative and positive electrical charges Source: own author

Heat can cause particles associated with the electron graviton to vibrate and associate with other neutrinos. The electron spin guides the magnetic field and its interactions.

"Mass and movement are inseparable concepts and fundamentally replaceable for all matter" [6].

\section{THE MAGNETIC FIELD}

The magnetic field, because it has associated particles, would not deform and would promote repulsion when the fields were approached. If they were simply magnetic waves, without any associated particles, the vectors would be in all directions and there would possibly be no magnetic repulsion.

It is possible that the particles in the magnetic fields have spin and very high speed of rotation - just like electrons. Because they have spin, they also promote electric field. The difference between electric and magnetic fields is in the form of density distribution in the elementary string and its internal and rotational movements. However, they must not have kinetic energy that would change the quantum state, as this would generate instability in the field. This magnetic field alone is "masked" by the total magnetic field; associated with gravity, the field particles become much more cohesive. Because they are the same size, the gravitational action is limited and the particles of the periphery and nucleus of the photon would not get close.

The gravitational interaction generated by the photon of the lower frequency electromagnetic radiation continues and is also shielded. Therefore, several types of electromagnetic waves can coexist simultaneously. Wave interference in air and vacuum can occur, but not destruction. 


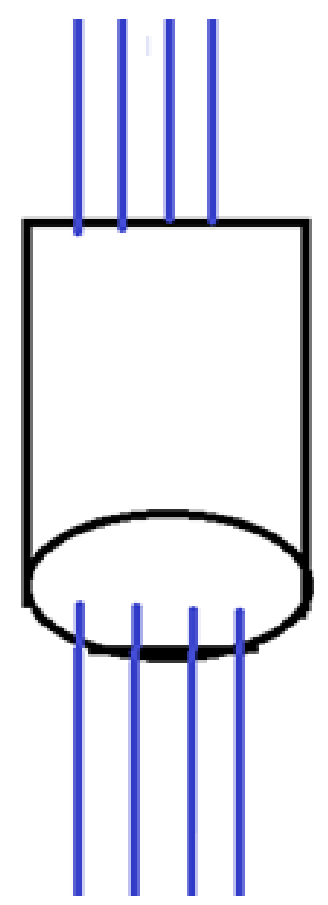

A unidade de campo magnético atrai outra unidade; o pólo norte se "acopla" ao pólo sul como uma espécie de encaixe. Campos iguals aproximados promovem repulsðo; esta, quando mais intensa, promove a energia potencial elástica nos magnetos. $\mathrm{E}=\mathrm{kx} \mathrm{x}^{\wedge} 2 / 2$

$0 x$, em metros, pode ser medido através da diferença entre a posiçăo inicial dos ímãs $e$ sua posição final.

ké a constante elástica.

A particula magnética pode, teoricamente, ser um neutrino; ele é acoplado ao grávíton.

O magnetismo entre as particulas mais a interaçăo magnética promove linhas de força magnéticas coesas, estáveis. As particulas magnéticas podem ser ligar também gravitacionalmente a outras $\mathrm{e}$ "instruir" a açào delas.

Only high frequencies cause interactions with organic matter because one photon after another behaves similarly to a solid and transmits a lot of stored energy in its fields.

\section{SPIN OF PARTICLES}

Electrons have a spin other than 1. This means that they have their own intrinsic motion. Similar to tornadoes, these promote a flow of neutral particles, which, interconnected by "strings", in a quantum entanglement, are responsible for the magnetic field; these magnetic "strings" come out of the electron "funnel" and lean perpendicularly to go towards the other pole and continue to interact with the other magnetic particles.

\section{INTERACTION BETWEEN PARTICLES IN ELECTROMAGNETIC WAVES}

When particles vibrate in a very fast "back and forth" movement, they have the same internal rotation, size and mass, have strong magnetic attraction and form smaller particles that are carriers of this magnetism, which can also be pulsatile, in a kind of "cord" . Waves with different vibration frequency and energy do not interact; they do not even reach the "center", because the outermost waves have higher frequencies and energies at shorter wavelengths, which means that the matter that has internal energy of intrinsic rotation, reflects some waves and absorbs others and that have equal frequency.

The waves pass between the molecular bonds; if they have high energy, they can ionize atoms, as in RX. Low frequencies, on the other hand, when emitted in the form of AM transmission, lose their strength as they penetrate the medium and the material, until they fall apart. Transmission power is also an important factor in reaching electromagnetic waves.

\section{ENERGY INTERACTION IN THE LIVING ORGANISM}

When electrical pulses are produced directly on the skin, there is no need for very high powers; a medium or high enough voltage is enough to promote the propagation of electric current in the body segment. This same current also promotes magnetic effects. The electrons and ions moved by the electric current deliver small "packages" of gravitational energy, more electric and magnetic, contributing to the formation of ATP.

During electrostimulation, electrons react with water molecules on the cathodic side to produce hydroxyl ions ($\mathrm{OH})$, while on the anodic side, protons $(\mathrm{H}+)$ are formed. Thus, between the anodic and cathodic interface, a gradient of protons and a potential gradient across the tissue and the medium is created. As a result, the protons, under the influence of the electric field and the difference in concentration, must move from the anode to the cathode. "As long as the ratio of proton formation at the anode interface is equal to the ratio of proton consumption at the cathode interface, the $\mathrm{pH}$ of the system (medium and tissue) remains without interference. When proton migration reaches the mitochondrial membrane $\mathrm{H}+$ ATPase, ATP will be formed. The oxidation of substrates, 
which is accompanied by the migration of protons through membranes, can also be electrically stimulated by the induced current of protons, activating a feedback process. Studies have shown that the use of microcurrents at 500 amps increases ATP production by up to $500 \%$, which increases the transport of amino acids, and these two factors contribute to an increase in protein synthesis.

The human body emits infra - red waves, in addition to reflecting light spectra. This is due to the organization of the coarse, corporal matter. But at the level of synapses the frequency is lower.

High frequencies are useful, when combined with low frequencies, to transport and deliver low frequency.

\section{COMBINATION OF ENERGY}

The combination of energy results in a single, interlacing particle. It would be like an "address" that involves magnetism on a minimal scale, along with electromagnetic and purely magnetic oscillation frequencies. The pure magnetic field tends to make the electromagnetic wave "rotate", with different speeds, direction of rotation, similar to an engine.

This connection tends to pass through the grotesque matter, because the encapsulations by intrinsic rotation and frequencies do not allow interaction with the atoms.

At the quantum level, entanglement, combination of energies or even brief interruptions are possible.

A new possibility of energy form is in frequencyshaped solenoids; if this is true, some characteristic of the particles could be transmitted to this waveform. Possibly other material solenoids could "pick up" frequencies, energy and if mechanical manifestations occur, the particles' own movements. Perhaps there would be an increase in mass of the group.

Each molecule has its own vibration frequencies, mainly dependent on the frequencies of the atoms that compose it.

An enzyme, due to its quaternary structure, has different frequencies in different fragments. These frequencies interact with electric fields in the valence layers and "increase" the electrical component next to the electron and the magnetic component next to the atomic nucleus. Molecules that have a three-dimensional structure whose atoms align with the functional of the enzyme receive this electromagnetic energy and react with another that also receives it. Then, chemical bonding occurs without energy consumption and enthalpy variation.

\section{CONNECTIONS THAT EXCHANGE HEAT DUE TO THE NEUTRINE FIELD}

The heat, in an endodermic reaction, promotes a greater agitation of the electrons in the valence layer. This heat is carried by neutrinos, which "migrate" towards the electrons. The masses of neutrinos between electrons act gravitationally; due to the difference in mass between the two being insignificant, there is a small gravitational force and consequently a physical separation. The greater the action of the gravitational force, the more the atoms become cohesive.

When atoms are linked together gravitationally, heat easily destabilizes the system by promoting kinetic energy of the electrons in the valence shell; these bonds break - if the thermal energy is transmitted by the electron neutrinos.

Mass and energy must go together. It is difficult to imagine one without the other. Without coupled energy, the mass becomes an isolated element and does not interact with others. There would therefore be no different connections and states of matter.

\section{NATURE OF THE ELECTROMAGNETIC IRRADIATION PHOTON}

The photon that detaches from the accelerated electron in the metallic object, like an antenna, carries the magnetic and electrical field components with it. In order for these fields not to fall apart, it is necessary that "something" keeps them in orbits; this something could be the gravitational pull and the neutrino field. But for it to occur, two bodies are needed; one would be in the nucleus of the photon, and the other in the orbit of its magnetic or electric fields. 


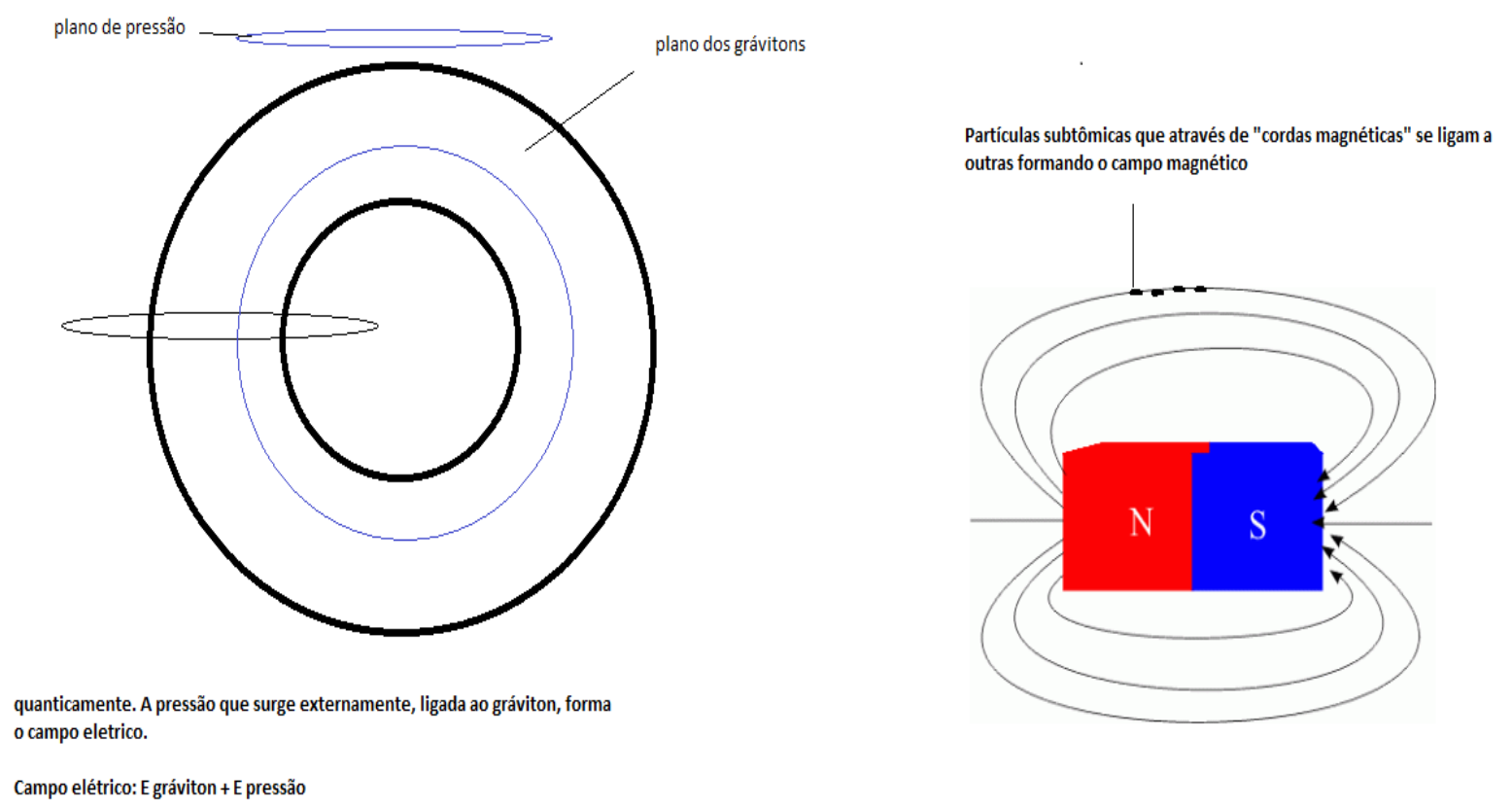

Fig 5:- Electric and magnetic fields

Source: own authorship

The particles associated with the magnetic and electric fields of the photon of the electromagnetic wave perform a kind of "shielding" to other electromagnetic waves; so they don't cancel out.

It is also for this reason that electro-magnetic waves are able to pass through walls-the particles of the fields do not "let" chemical interactions occur. They form a kind of armor.

It is likely that, to maintain the gravitational and electric fields, the volume of the photon will be the same as that of the electron. However, according to quantum mechanics, it is not possible with current means to measure the volume of the electron and consequently the photon.

Theoretically, to keep the neutrinos inside and close, the photon brings with it pure elements of gravity. For energy conservation to occur and the wave to have a wide range, it is necessary that the kinetic energy of the photon is fed back; this is possible due to the neutrinos in the nucleus.

In an electrically conductive medium, when the electron moves in one direction, the direction of movement of the electric and magnetic fields is one; when it moves in the opposite direction, the direction is the opposite.

The greater amplitude of the wave, produced by the greater power of the emitter of electromagnetic waves, causes the energy of the electric field to "capture" more neutrinos. Their high kinetic energy is not canceled out and part of it is used to move the photons in one direction.

That is why higher power radio transmitters have a longer range.

To transmit heat, it is necessary that neutrinos have high kinetic energy.

The mass of the neutrinos is so small that it does not even change the measurement values of the energy of the electromagnetic wave even with the current apparatus and specific procedures. The most that has been achieved to date is to prove that quantum components of waves of very high frequencies produce a second harmonic. According to the arguments of this article, the neutrino field can be considered these quantum components

The electric field of the photon is also a "part" of the electric field of the electron and comes off when the electron is agitated. The same is true of the magnetic field. In other words, the photon of the wave is able to induce and carry replicas with magnetic and electric fields; this is only possible if matter, together with energy, participates in this process.

Electromagnetic wave of different frequency, since produced together with the wave of higher frequency, has its photons interacting through gravitons of the second photons. The former also have gravitons; but they do not "touch" due to the similar gravity and the kinetic energy of the photons, which predominates. 


\section{CALCULATION OF TOTAL ENERGY FROM ELECTROMAGNETIC WAVE AND NEUTRINE FIELD}

The mass of neutrinos interacts with the gravitons present in the electromagnetic wave; the particles of the gravitons stay inside the photon and promote bonds with the neutrinos outside the photon and react with them gravitationally. Because they have greater kinetic energy, neutrinos outside the center of the photon are not attracted to it.

\section{Formulas:}

Etotal $=$ Eonda + Ecalor + Electric field + Magnetic field + Gravitational field + Eckinetics-neutrinos

Calculation of the neutrino field:

$(1 /$ micro0 $) * \mathrm{E} * \mathrm{~B}=\mathrm{hf}+\bullet \gamma \max .576+1 / 2 \varepsilon 0 \mathrm{E}^{2}+\mathrm{B}^{2} / 2 \mu 0$ + G.m1.m2 / r + mneutrinosv² 2

mneutrinosv $\mathbf{2}^{2} \mathbf{2}=\mathbf{( 1 / \mu \mathbf { 0 } )} * \mathbf{E} * \mathbf{B}-\bullet \gamma \max .576+1 / 2 \varepsilon 0 \mathrm{E}^{2}$ $+\mathrm{B}^{2} / 2 \mu 0-$ G.m1.m2 / r

Ecineeticaneutrinos $=\Delta \mathrm{aU}$

delta $\mathrm{U} /$ deltaadeltat $=(1 / 2) * \mathrm{c} * \mathrm{e} 0 * \mathrm{E} 0^{2}$

Total density of stored energy: $\varepsilon 0=\mathrm{E}^{2}$

$\mathrm{E}=$ electricity

Densities of electrical and magnetic energy: $\mu \mathrm{E}(\overline{\mathrm{r}}, \mathrm{t})=1 / 2$ $\varepsilon 0 \mathrm{E}^{2} \quad$ e $\mu \mathrm{B}(\overline{\mathrm{r}}, \mathrm{t})=\mathrm{B}^{2} / 2 \mu$

Egravitational $=$ G.m1.m2 $/ \mathrm{r}$

And kinetics-neutrinos $=\mathrm{m}^{2} / 2$
Thermal energy: gamamax.5762

$\mathrm{h}=6.625 .10^{\wedge}-34(\mathrm{~J}$ s $)$ Plank constant

$\mathrm{k}=1,381.10^{\wedge}-23(\mathrm{~J} / \mathrm{K})$

\section{FLOW OF SOLAR ENERGY}

The Earth-Sun distance is $1 \mathrm{AU}=1.5 \mathrm{x} 1011 \mathrm{~m}$ Therefore, the energy flow (energy per unit time per unit area) that reaches the Earth's surface is $F=L /(4 \pi d 2) F=$ $1379 \mathrm{~W} / \mathrm{m} 2$

Classic example: the photoelectric effect. Light falling on a metal can cause an electron to be ejected from it (photoelectric cells are based on this principle). This effect can only be understood if the light is treated as a particle: quantum of light or energy = photon.

The photon energy is proportional to the frequency of electromagnetic radiation: energy $=$ frequency $\times \mathrm{h}$ or $\mathrm{E}=\mathrm{h}$ $v$ where $\mathrm{h}$ is the Planck constant: $\mathrm{h}=6.62607 \times 10-34$ joules $\times$ second.

A prism is capable of separating white light in the colors of the rainbow What differentiates each color. It is its frequency and wavelength.

Red: $\lambda=630-740 \mathrm{~nm}$ Orange: $\lambda=590-630 \mathrm{~nm}$ Yellow: $\lambda=560-590 \mathrm{~nm}$ Green: $\lambda=500-560 \mathrm{~nm}$ Blue: $\lambda=$ 480-500 nm Anil: $\lambda=440-480$ nm Violet: $\lambda=380-440 \mathrm{~nm}$

Each band of the electromagnetic spectrum is given a different name, according to its properties. For example, electromagnetic radiation with a wavelength between 380 and $740 \mathrm{~nm}$ is called visible light [1].

\section{Comprimento de onda $(\lambda)$ - em metros}

\begin{tabular}{ccccccc|c|}
\hline Radio & Microond. & Infraverm. & Visivel & Ultraviol. & Ralos-X & Ralo Gama \\
\hline $10^{3}$ & $10^{-2}$ & $10^{-5}$ & $5 \times 10^{-6}$ & $10^{-8}$ & $10^{-10}$ & $10^{-12}$
\end{tabular}

Do tamanho de...
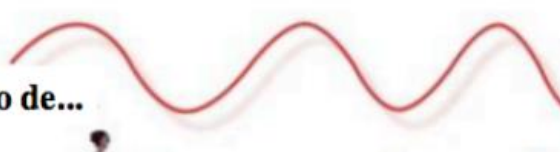

ح
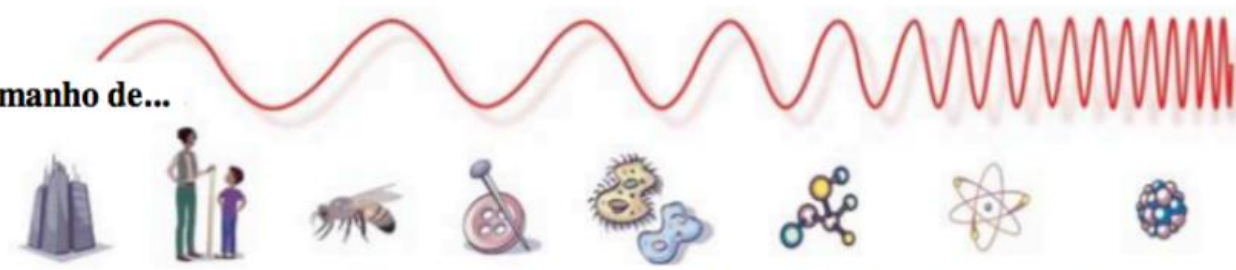

prédios humanos abelha agulha protozoários moléculas átomos núcleo atomico

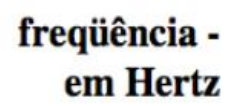

Temperatura
- em Celsius
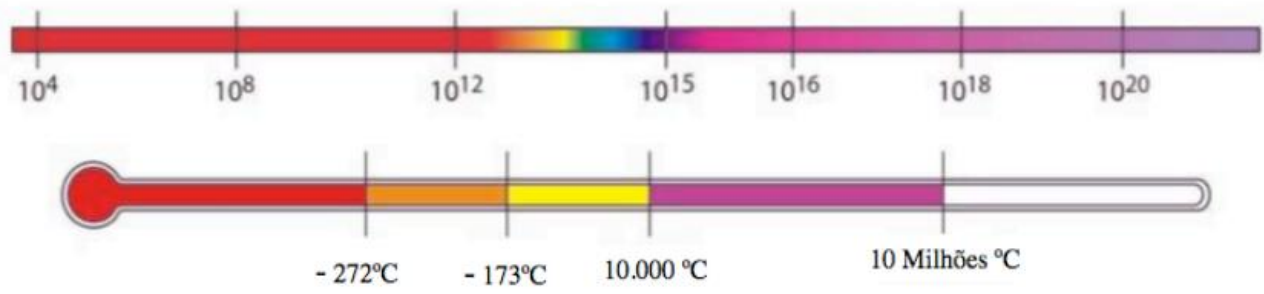

Fig 6:- Spectrum of electromagnetic radiation

Source: general secretary [1] 


\section{CONCLUSION}

The forms of energy are apparently independent and have different effects. When considering a theory based on strings, filaments of matter, one can explore possibilities of interactions inside and outside this element, which form different types of energy.

In the first half of 2020 the phenomenon of the second harmonic in superconductors was evidenced. The theory that a neutrino field may exist in association with photons from electromagnetic waves may explain the emergence of this harmonic as a direct result of this field; in low frequency waves, it would not have enough matter and energy to cause the observed effect. In addition, it can also explain the photon wave particle duality; according to the theory presented, the photon presents matter when associated with neutrinos, which contribute to the propagation of the electromagnetic wave.

\section{REFERENCES}

[1]. general secretary. What is 'forbidden light', a discovery that could revolutionize quantum physics. 2020. Available at <https://asmetro.org.br/portalsn/2020/05/27/o-que-ealuz-proibida-descoberta-que-pode-revolucionar-afisica-quantica/>. Accessed 04 Jun 2020.

[2]. What is light? Available at < https://edisciplinas.usp.br/pluginfile.php/135090/mod _resource/content/1/Mono_Material.pdf.>. Accessed May 27, 2020.

[3]. [3] Electromagnetic waves. Available at <https://www.slideserve.com/barid/ondas-eletromagnticas>. Accessed 05 Jun 2020.

[4]. Electromagnetic waves. 2002. Available at <http://midia.cmais.com.br/assets/file/original/e4ab79 cf4cfa5e18283eb5b1146e3740abf19bbe.pdf>. Accessed May 26, 2020.

\section{[5]. Photon energy.Available} $<$ https://en wikipedia org/wiki/Energia_do_t/ ton>. Accessed 04 Jun 2020.

[6]. Juliana Fernandes de Almeida. Microcurrent in Aesthetics. Available inhttps://www.portaleducacao.com.br/conteudo/artigo s/idiomas/microcorrente-na-estetica/5621. Accessed 04 Jun 2020. 